J. Male and J. R. Prior (London) also reported the first examples of fluorescent emission in the ultra-violet from diamond at energies near $5 \cdot 1$ and $5 \cdot 3 \mathrm{eV}$.

The growing interest in silicon carbide was shown by four papers on practical aspects involving various mechanical properties such as friction and wear of diamond and silicon carbide, by C. Brookes, by D. R. Miller, by L. M. Fitzgerald and by F. Field and N. L. Hancox, all from Cambridge. The steady improvement in the purity control of silicon carbide was described by W. F. Knippenberg (Holland). Using as starting material cubic silicon carbide prepared by decomposition of chlorosilanes, the total content of foreign atoms, which were mostly chlorine, could be reduced to less than $10^{16} \mathrm{~cm}^{-3}$. Using such pure material, H. Daal, W. Knippenberg and J. D. Wassher measured the Hall constant and the resistance of silicon carbide from $80^{\circ}$ to $1,400^{\circ} \mathrm{K}$. Much lattice imperfection is indicated since the number of acceptors and donors exceeds the number of chemical impurities by an order of magnitude. Occupying as it does a key intermediate position between silicon and diamond, all the properties of silicon carbide are of great interest and importance. However, because of the different possible atomic arrangements such as cubic and such polytypes as $4 \mathrm{H}$ and $6 \mathrm{H}$ in the hexagonal form, the interpretation of the optical and electrical properties of silicon carbide is complex. D. Hamilton, W. J. Choyke and L. Patrick (United States) have examined the optical absorption and photoluminescence of $4 \mathrm{H}$ silicon carbide, extending earlier work on the $6 \mathrm{H}$ polytype. A characteristic edge luminescence was observed in the pure $4 \mathrm{H}$ crystals and parts of the spectrum were found to have photon energies greater than the energy gap of polytype $6 \mathrm{H}$. Corresponding absorption experiments showed that the $4 \mathrm{H}$ energy gap exceeds the $6 \mathrm{H}$ energy gap by $0.25 \mathrm{eV}$. The transitions are indirect and exciton absorption is observed.
The impression gained from the Conference was that although diamond physies is still in its infancy. sufficient experiments have now been performed over a wide range of properties and techniques to indicate that a relatively few well-chosen experiments, such as the investigation of radiation damage close to the threshold energy, should throw real light on fundamental problems in solid state physics. As Prof. R. W. Ditchburn pointed out at the beginning of the Conference, the unique property of diamond is its stability and the simplicity of its composition. These striking advantages compared, for example, with the alkali halides, as set out in a conference on crvstal luminescence held two weeks earlier in Turin. seem to me far to outweigh the rolative intractability of diamond as a material in that it prevents the overwhelming flood of experimental data which would undoubtedly ensue if simple methods of 'doping' diamond were available. Diamond physics is on an orderly march which should lead to a real advance in our understanding of solid state physies.

Advantage was taken of the opportunity provided by the Conference to present the Duddell Medal to Prof. R. V. Jones, who then spoke on some developments of the optical lever. In an excellent lecture, enjoyed by all, Prof. Jones described in particular the development of the optical lever in the past ten years up to the present stage when the performance approaches the limit set by the statistical fluctuations of photons in the light beam. Applications covered the evaluation of the thermal fluctuation limit for galvanometers, the detection of the change of linear momentum in a beam of light after refraction, and the development of a sensitive infra-red detector based on linear thermal expansion. The method failed to detect any deviation of a beam of light in an inhomogeneous transverse magnetic field of mean value 8,000 oersteds, although a change in the velocity. of light of about $70 \mu \mathrm{sec} .^{-1}$ should have given a devia. tion equal to the r.m.s. error.

\title{
THERMAL MOTION IN CRYSTALS AND MOLECULES
}

$\mathrm{T}$ HE fifth international congress of the International Union of Crystallography which was held recently in Cambridge was followed by two symposia, one of which was devoted to papers on Thermal Motion in Crystals and Molecules. This symposium lasted for two days, August 22-23, apart from an introductory lecture which was given during the main congress in the previous week. It consisted of five invited papers and thirty other contributions, abstracts of which had been published in advance of the meeting by the International Union of Crystallography.

In the early days of crystallographic studies by $\mathrm{X}$-ray diffraction techniques, thermal motion was a disturbing factor for which rather empirical correction had to be made, by means of the familiar DebyeWaller theory, before the crystallographer could make a crucial comparison of his experimental intensity results with deductions from any postulated structural model. Since then the increasing accuracy of measurements and ease of interpretation have permitted the process to be largely reversed and experiment now allows a detailed assessment of atomic motions to be made and analysed into various types of vibration. Investigators are no longer restricted to the use of X-rays for electrons, and neutrons are now being widely used in support. Moreover, further theoretical work on the various scattering processes and improvements in experimental techniques have played their parts in increasing the interest in this field of work. During the past few years it is particularly noteworthy that rather empirical descriptions of atomic motions are being superseded by much more comprehensive descriptions of co-operative motions of molecules and crystals as a whole. To a considerable extent, interest has been fostered by the increasing availability of computing machines and programmes able to analyse the experimental results in terms of the many parameters needed for a full description of thermal motion.

Although receptive of any aspect of thermal motion in crystals and molecules, the symposium was aimed at providing discussion of three particular topies: first, the study of the elastic and inelastic scattering of $\mathrm{X}$-rays and neutrons by solids, as a 
source of information on the amplitudes and frequencies of motion in crystals and their dependence on temperature; secondly, the use of electron diffraction measurements to study molecular motion in gases; and thirdly, data on residual motion in crystals at low temperatures. The introductory lecture by Prof. J. C. Slater served as a general lecture to the whole congress and was entitled "The Interaction of Mechanical and Electromagnetic Waves in Crystals". While summarizing the more conventional aspects of the scattering of radiation by phonons in crystals, this lecture surveyed the interactions over a very wide range of frequency, and included an account of recent work on the explanation of ferroelectric transitions from the point of view of lattice dynamics. The treatment of Cochran, whereby a ferroelectric transition is emphasized as being a limit of stability against a particular mode of vibration, was shown as a development of the much earlier work of Huang and others on anomalous dispersion in the infra-red region, where a transverse electromagnetic wave interacts with transverse mechanical oscillations in a crystal.

The main symposium began with an invited paper by Dr. D. W. J. Cruickshank, who discussed, chiefly from a theoretical point of view, the determination of atomic vibration amplitudes from $\mathrm{X}$-ray diffraction data, particularly in molecular crystals where the motions are anisotropic. For each atom a vibration tensor with six components can be deduced and these tensors can then be combined to give a picture of both rigid-body vibrations of the molecule as a whole and of internal vibrations within the molecule. At room temperature the latter are relatively small, but they become of greater importance as the temperature is reduced. It was emphasized that $\mathrm{X}$-ray diffraction data alone are not sufficient to give an unambiguous solution, and it is necessary to employ spectroscopic evidence as well in order to distinguish, for example, between internal twisting vibrations and angular oscillations as a rigid body. Investigations concerning the perchlorate ion in perchloric acid monohydrate $\left(\mathrm{H}_{3} \mathrm{O} . \mathrm{ClO}_{4}\right)$ and of ethane were described as examples of the essential part played by spectroscopic data in the analysis of the various motions. The following, complementary, paper by Dame Kathleen Lonsdale surveyed the problems from a more practical point of view in the light of much experimental information accumulated by the author and her colleagues at University College, London. In practice, before true thermal vibrations can be studied, allowance has to be made for zero-point energy, structural disorder and bonding anisotropy. Measurements at reduced temperature and parallel studies with X-rays and neutrons may be necessary in order to secure unequivocal results. The partition of the total thermal motion among internal and rigid-body vibrations was discussed, taking urea, hexamine, naphthalene and anthracene as examples. In the latter case where the directions of the principal axes of atomic vibration are not fixed by crystal symmetry, wide discrepancies in their specification from different, seemingly accurate, sets of results were found. It would seem to be well established that there is still a considerable need for data with improved accuracy, more free from both random and systematic errors, if the detailed conclusions which are drawn from structural analyses of this type are to be justified.

These two general review papers were followed by several descriptions of structural analyses which depended on the principles previously enunciated, illustrating in the main the relation between atomic motion and the environment of an atom, both within its own molecule and among its neighbours, and with some attention being directed to developments of experimental technique. Increasing attention is certainly being given to measurements at low temperature, but there are still only a few substances for which a detailed analysis has been made at more than one temperature. The value of supporting evidence from other physical methods of analysis, such as specific heats, nuclear magnetic resonance and spectroscopy, is very great.

Both Dr. Cruickshank and Dame Kathleen Lonsdale referred to the information on molecular motion which could be obtained from work on the diffuse inelastic scattering, as distinct from the elastic Bragg peaks, and this theme was taken up in more detail by later contributors. Papers by Dr. W. Cochran and Prof. W. Hoppe described the theoretical calculation of the diffuse scattering from molecular crystals, pointing out the relative significances of the displacements due to translation and rotational motion, and distinguishing between the enhancement of diffuse scattering in the neighbourhood of reciprocal lattice points and that over extended areas. Several succeeding contributions described the experimental verification of these ideas and their value in structure determination. A paper by Dr. M. L. Canut illustrated how experimental measurements of the shape and location of the extended regions of diffuse scattering were in good agreement with calculations made from the shape and orientation of the molecules for substances such as hexamine, pentaerythritol and naphthalene. A related contribution from Drs. E. Sandor and W. A. Wooster emphasized that the detailed interpretation of the diffuse scattering might be carried out accurately enough to be of value in structure analysis. Particularly for planar molecules, the orientation in the structure of a molecule of known shape could be predicted and a molecular. Patterson function could be calculated.

In a group of papers on electron diffraction work on gases, it was evident that the greatly increased experimental accuracy which can now be attained has stimulated interest in more detailed work on the diffraction process and the force fields in molecules. Prof. Y. Morino discussed the exact relation of the experimentally determined quantities - the mean atomic distance and the mean square vibration amplitudes-to the actual equilibrium distance and the detailed molecular field. Prof. O. Bastiansen and his colleagues showed that certain linear molecules may appear to be bent on account of vibrations perpendicular to the bond, because the longer interatomic distances within the molecule appear to be shorter than expected from a summation of observed bond distances. Carbon suboxide was shown to be a case where it is difficult to distinguish between a linear molecule with large out-of-line vibrations and a bent equilibrium configuration. One was impressed by recent improvements in experimental techniques; for example, $K$. Hedberg described how it was now possible to measure the increase with temperature of the vibration amplitudes in phosphorus trichloride, in fair agreement with predictions from spectroscopic data. In discussions of these electron diffraction papers, attention was directed to the importance of using complex scattering factors, reliable values of which are now becoming known and listed. 
The influence of atomic vibrations on measured values of lengths of bonds is not, of course, limited to electron diffraction work, and a paper by $\mathbf{H}$. Levy discussed this problem in relation to precise structure refinements by X-ray and neutron diffraction methods. It was shown how the apparent interatomic distance for a pair of atoms depended on whether or not their individual thermal motions were linked or independent. Any attempt to deduce the equilibrium distance of separation, as distinct from the centroid separation, depends on reaching a precise knowledge of the atomic vibrations after making measurements at various temperatures, followed by an extrapolation and allowance for zero point motion. In discussion it was made clear that whenever corrected distances are given it is very essential to specify on what assumptions the corrections have been made. The effects are of particular importance when the motions of hydrogen atoms are being considered and are of especial interest in work on neutron diffraction. This paper, in fact, formed part of a session which was devoted to the contribution of neutron methods to our knowledge of thermal motion, which Dr. W. Cochran opened with a discussion of the great value of measurements of energy distributions of scattered neutrons in a review of recent work on lattice dynamics by neutron spectroscopy at Chalk River. Of particular interest were an extensive series of precise measurements of the dispersion relation, between frequency and wave-number, for the acoustic vibrations in single crystals of lead. In order to interpret the results it is necessary to assume that long-range forces are important in the solid and it is shown that their range becomes less as the temperature is increased. From the widths of the peaks in the neutron-energy spectrum it is possible to deduce the life-time of the phonon vibrations. In a further comprehensive set of measurements the lattice dynamics of the alkali halides and of lithium hydride were investigated. As an example of the contribution of neutron methods to disentangling the effects of thermal vibration and bond anisotropy, a paper given by Dr. R. S. Calder described concurrent neutron and $\mathrm{X}$-ray work on lithium hydride.

In the final session of the symposium, Prof. I. Waller described some recent theoretical work on the frequency distribution and dispersion relations for alkali halides at low temperatures, with particular reference to the approximations which have been made in calculating the form of the zero-point energy vibration amplitudes. The conclusions are in good agreement with results of diffraction experiments. In an interesting survey of a wide range of experimental data, F. H. Herbstein examined the values of
Debye temperature determined for cubic crystals by methods such as specific heat and elastic constant measurement, as well as by diffraction studies at one or several temperatures. At room temperature there is reasonable agreement between different methods, but at low temperatures such meagre $X$-ray data as exist suggest that the apparent $\Theta$ values may be lower than those found by other methods. It would seem that some accurate and comprehensive X-ray work in this field would be rewarding.

Two papers were given on the contribution of nuclear magnetic resonance to studying thermal motion. In a review of this application, Prof. E. R. Andrew emphasized that whereas previous speakers had been concerned solely with 'vibrations', there were other forms of motion, such as hindered rotation and self-diffusion, which only occurred infrequentlythough involving much greater movements-and which could not be examined by diffraction techniques. Examples were given of how these topics can be investigated quantitatively by nuclear magnetic resonance techniques and how the postulated effects on the spectrum can also be demonstrated by absorption measurements with rotating crystals. Dr. J. A. S. Smith described an application of nuclear magnetic resonance methods to the examination of single crystals of urea and thiourea which demonstrated the onset of hindered rotation about the $\mathrm{C}=\mathrm{O}$ and $\mathrm{C}=\mathrm{S}$ bonds in the two cases. As a result the molecules flip between two possible equilibrium configurations, giving rise to large apparent temperature factors for some of the atoms as judged by $\mathrm{X}$-ray diffraction work.

In conclusion, it may be worth recording two personal impressions which remain from the papers and discussions. First, although the precision of experimental measurements in these investigations is very high, there still remain some significant gaps in interpretation which leave room for systematic errors and may make some of the apparent accuracy illusory. Secondly, in spite of the increasing identity of equip. ment used over the world, one can still be struck by the individuality of thought and methods of approach which characterizes the different schools of investigators.

The symposium was attended by about 400 persons, mainly accommodated in the large lecture theatre of the Arts School at Cambridge, but with an over-flow in the adjoining Cavendish Laboratory served by a closed-circuit television set. It was perhaps significant that even for the less-crowded sessions there were many who preferred the quieter, airy contemplation of the television screen to the hurly-burly of the lecture room.
G. E. BACON

\section{THE INTERNATIONAL SCIENTIFIC FILM ASSOCIATION}

$\mathrm{T}$ THE fourteenth annual congress of the International Scientific Film Association was held in Prague during September 16-24. It was attended by 120 delegates and observers from twenty-four countries. The British delegation of twenty people was led by Mr. Edgar Anstey, president of the Scientific Film Association. Associated with the congress was the Third Festival of Films presenting Science.

The International Scientific Film Association is a non-profit-making and non-governmentalorganization, officially recognized for consultative status with Unesco. Membership is open to any organization representative of the science film movement in the country of that organization.

The congress were welcomed officially by the Czechoslovak Minister of Education and Culture, Dr. Frantisek Kahuda, and by the managing director of Czechoslovak Film, Mr. Alois Polednak.

The Czech Organizing Committee-led by Dr. Vladimir Vaclavek-provided a delightful centre in 